\title{
Sleep apnoea and cancer: the new challenge
}

\author{
Patrick Lévy ${ }^{1,2,3}$, Diane Godin-Ribuot ${ }^{1,2}$ and Jean-Louis Pepin ${ }^{1,2,3}$ \\ Affiliations: 'Univ Grenoble Alpes, HP2 Laboratory, Grenoble, France. ${ }^{2}$ INSERM, HP2 Laboratory U1042, \\ Grenoble, France. ${ }^{3}$ University Hospital, CHU Grenoble, Physiology Department, Grenoble, France.
}

Correspondence: Patrick Lévy, EFCR, CHU Grenoble, 38043 Grenoble, France. E-mail: PLevylachu-grenoble.fr

-

@ERSpublications

Epidemiological studies suggest that excess mortality in OSA patients is partly related to a higher risk of cancer http://ow.ly/vBNY5

Obstructive sleep apnoea (OSA) is a well-known public health problem owing to its high prevalence and the numerous consequences of the disorder, including excessive daytime somnolence, cognitive impairment and consequently traffic accidents [1]. There is an excess in cardiovascular mortality that has been repeatedly reported in longitudinal cohorts, in both general and clinical populations. Despite many confounding factors, including age, sex and obesity, evidence has accumulated over the past 25 years regarding strong associations between OSA, cardiovascular diseases i.e. hypertension [2], coronary artery disease, cerebrovascular disease, heart rate and conduction disorders, and excess mortality. Through a complex interaction between obesity, metabolism, sleep and sleep apnoea, metabolism seems to be altered by OSA [3], although it remains uncertain whether treating OSA may improve blood glucose control, lipid metabolism [4] or adipose tissue distribution [5]. More recently, it has been suspected that the excess in mortality in OSA could not only be attributed to cardiometabolic consequences but also to cancer. Cancer has been found to be associated with sleep disordered breathing (SDB) in two large observational studies $[6,7]$. This had been previously suspected through proof-of-concept animal studies that demonstrated an association between intermittent hypoxia, a major consequence of SDB, carcinogenesis and acceleration of tumour growth [8]. Intermittent hypoxia is a particular condition with relatively specific cellular effects [9], including changes in host immune responses that could favour cancer progression [10]. The relationship between SDB and cancer has been studied in animal $[8,11,12]$ and clinical studies; they suggest that SDB, mainly through intermittent hypoxia, is likely to be associated with an increase in growth rate [8], incidence [6] and mortality [7] of cancer. In addition, sleep fragmentation, another hallmark of sleep apnoea, has also been demonstrated to promote tumour progression in animal models through recruitment of tumourassociated macrophages and Toll-like receptor 4 signalling pathways [13].

In this issue of the European Respiratory Journal (ERJ), MarTínez-GARCía et al. [14] analysed the relationship between the severity of SDB and the aggressiveness factors of cutaneous malignant melanoma (CMM). They performed a multicentre observational study in 82 consecutive patients diagnosed with CMM, of whom 56 patients were finally included in the study. Measurements of melanoma aggressiveness included: tumour mitotic rate, Breslow Index, presence of ulceration, stage of disease and growth rate of melanoma. The severity of SDB, i.e. apnoea/hypopnoea index (AHI) and desaturation indexes, was correlated with melanoma aggressiveness markers. There are several limitations in this study. Age is a major confounder since, as mentioned by the authors, it will both favour melanoma evolution due to persistent exposure to aetiological factors and the occurrence of SDB. The sample size is relatively small. The prevalence of sleep apnoea is surprisingly high in this middle-aged population ( $>30 \%$ with an AHI $>30$ events $\cdot \mathrm{h}^{-1}$ ). Some confounders were not accounted for in this relatively small population. For example, tobacco and alcohol consumption are common risk factors for both sleep apnoea and melanoma and might

Received: April 072014 | Accepted: April 072014

Conflict of interest: None declared.

Copyright @ERS 2014 
have, in part, supported the correlation between SDB and melanoma. The data on CMM growth rate, which depended on patients recall, were somewhat subjective and the diagnosis of CMM was retrospective. Nevertheless, it demonstrates that SDB severity is likely to be associated with aggressiveness of CMM. This will need to be further confirmed in larger clinical cohorts.

Metastatic cutaneous melanoma accounts for the majority of skin cancer deaths due to its aggressiveness and high resistance to current therapies [15]. UV exposure and melanoma are causally related [16]. Recent data showed a direct mutagenic role for UV light in melanoma pathogenesis [17]. There is a persistent rise in global incidence with 160000 new cases per year and 48000 deaths. The main reason for this could be increased exposure of pale white skin to natural UV radiation [16]. The latent period from initiation of melanoma carcinogenesis to clinical presentation can be decades, which probably explains why the incidence rates are still increasing in most countries. Melanoma cells can use different migratory strategies, depending on environment, to exit the primary tumour mass and invade surrounding and later distant tissues [15]. One of the major mechanisms is neoangiogenesis. Melanoma cells have been reported to secrete a number of angiogenic growth factors such as vascular endothelial growth factor (VEGF), placenta growth factor, basic fibroblast growth factor, transforming growth factor (TGF)- $\alpha$ and TGF- $\beta$, interleukin- 8 and platelet-derived growth factor-B [15]. Several studies have shown a positive correlation between melanoma neovascularisation and poor patient prognosis, overall survival, ulceration and higher rate of relapse [18].

The link between OSA and intermittent hypoxia with cancer development has been very recently discovered. Few experimental studies have been published so far, but all have confirmed that intermittent hypoxia plays a pivotal role in the cancer promoting effect of OSA, although there is scant information on the pathophysiological mechanisms involved. Interestingly, most experimental data have been obtained using melanoma tumour models $[8,11,12]$, adding additional strength to the clinical study published in this issue of the ERJ.

\section{What are the mechanisms linking sleep apnoea and cancer development?}

Cancerous tumours are characterised by proliferation of a cellular clone with unlimited proliferation capacity, associated with abnormal angiogenesis within the tumour environment. Interactions between the tumour and its microenvironment (endothelial cells and stromal cells, such as fibroblasts or macrophages) modulate tumour cell survival and proliferation. This triggers the development of a new vascular bed oriented towards the hypoxic tumour core. It also enhances metastatic tumour potential and resistance to anti-cancer drug treatment [19]. This is particularly due to physiology of the tumour microenvironment. Indeed, while tissue oxygen tension $\left(\mathrm{PO}_{2}\right)$ actually ranges between 10 and $80 \mathrm{mmHg}$ in different tissues, tumour $\mathrm{PO}_{2}$ varies between 2 and $10 \mathrm{mmHg}$ [20]. When adapting to this hypoxic microenvironment, cancer cells have to develop adaptive strategies mediated by the transcription factor hypoxia-inducible factor-1 (HIF-1). HIF-1 mediates adaptive (physiological) responses to hypoxia, such as erythropoiesis, angiogenesis and glycolysis, as well as maladaptive (pathological) responses in various disease states, such as cancer and OSA [21]. Major HIF-1 target genes are involved in angiogenesis, tumour development and metastasis [22]. Regarding angiogenesis, it is noticeable that blood vessel formation in the tumour microenvironment is progressive and irregular, resulting in spontaneous fluctuations in blood flow leading to temporary local intermittent hypoxia. In addition, it has been shown that these phases of hypoxia and reoxygenation contribute significantly to tumour growth, angiogenesis, chemoresistance and radioresistance $[23,24]$. In accordance with these in vitro observations, it has recently been confirmed that whole-body intermittent hypoxia, as seen in OSA, enhances cancer progression by promoting tumour growth, angiogenesis and metastasis $[8,12,24]$.

We, and others, have shown that rodents exposed to intermittent hypoxia express a sustained activation of HIF-1 that persists when the animals return to normoxic conditions $[25,26]$. This is in accordance with in vitro observations showing that intermittent hypoxia was a much more potent stimulus than sustained hypoxia for HIF-1 activation [27]. Also, HIF-1 activity rapidly decreases upon cessation of sustained hypoxia, whereas it remains elevated following intermittent hypoxia [28]. The mechanism behind the sustained activation of HIF-1, induced by intermittent hypoxia or OSA, appears to be the oxidative stress produced by the repetitive oxygenation-desaturation sequences. Indeed, OSA has been considered as an oxidative stress disorder [29]. It has further been confirmed that chronic intermittent hypoxia leads to important oxidative stress in various tissues [30-34]. The role of reactive oxygen species (ROS) in regulating HIF-1 activity is at least as important as hypoxia per se. Hence, intermittent hypoxia triggers NADPH oxidase-dependent ROS production, which activates phospholipase $\mathrm{C}$ leading to calcium-calmodulin kinase (CamK) and protein kinase C (PKC) activation. PKC stimulates mTOR dependent synthesis of the $\alpha$ subunit of HIF-1 and inhibits prolyl-hydroxylase-2 dependent HIF- $1 \alpha$ degradation. CamK also promotes the interaction between HIF- $1 \alpha$ and its co-activator p300, thereby leading to transcriptional activation. In contrast to continuous hypoxia, in which HIF- $1 \alpha$ is rapidly degraded (half-life $<5 \mathrm{~min}$ ) on reoxygenation, 
HIF- $1 \alpha$ levels remain elevated following intermittent hypoxia due to the persistent activation of mTOR [21]. This can explain persistent HIF-1 activity observed hours after termination of intermittent hypoxia or during the daytime in OSA, as recently reported by KACZMAREK et al. [35]. One fact that is particularly relevant regarding the potential mechanisms behind the results of the present study [14] is that KACZMAREK et al. [35] observed increased HIF-1 $\alpha$ expression in skin biopies from OSA patients.

Therefore, OSA could lead to enhanced and sustained HIF-1 activity both in the tumour and its environment, thus, promoting VEGF-related neovascularisation. This is supported by the increased plasma and tissue VEGF levels in OSA patients [35-38], as well as in animals exposed to intermittent hypoxia [39-42].

In summary, there is mechanistic evidence that the two main stimuli associated with sleep apnoea, i.e. sleep fragmentation and intermittent hypoxia, promote tumour growth and progression in animal models. Epidemiological studies in humans suggest that the excess mortality in OSA patients is at least partly related to a higher risk of cancer. The study of MARTínEZ-GARCía et al. [14] has some methodological limitations, but raises original and important questions in the field of melanoma and OSA. There is a disproportionate incidence of thick melanoma tumours in males aged $>60$ years [43], a population typically exposed to OSA for many years. By contrast, females have a better survival compared with males. Since females mainly develop OSA after menopause, this could be explained by less exposure to intermittent hypoxia before melanoma occurrence. For all these arguments, OSA should be considered as a potential novel risk factor for cutaneous melanoma. Overall, there is a need to extensively study the relationships between OSA and cancer of different origins. As suggested by PEPPARD and NIETO [44], SDB-cancer associations will be examined in existing study populations. In addition, investigations specifically designed to examine SDB-cancer associations, including the relationship of SDB, or SDB treatment, with cancer progression and prognosis in patients newly diagnosed with specific cancers are needed. A strong causal association between high SDB prevalence and carcinogenesis would have clear implications for preventing and managing SDB [44].

\section{References}

1 Strohl KP, Brown DB, Collop N, et al. An official American Thoracic Society clinical practice guideline: sleep apnea, sleepiness, and driving risk in noncommercial drivers. An update of a 1994 statement. Am J Respir Crit Care Med 2013; 187: 1259-1266.

2 Parati G, Lombardi C, Hedner J, et al. Recommendations for the management of patients with obstructive sleep apnoea and hypertension. Eur Respir J 2013; 41: 523-538.

3 Lévy P, Bonsignore MR, Eckel J. Sleep, sleep-disordered breathing and metabolic consequences. Eur Respir J 2009; 34: 243-260.

4 Pépin JL, Tamisier R, Lévy P. Obstructive sleep apnoea and metabolic syndrome: put CPAP efficacy in a more realistic perspective. Thorax 2012; 67: 1025-1027.

5 Sivam S, Phillips CL, Trenell MI, et al. Effects of 8 weeks of continuous positive airway pressure on abdominal adiposity in obstructive sleep apnoea. Eur Respir J 2012; 40: 913-918.

6 Campos-Rodriguez F, Martinez-Garcia MA, Martinez M, et al. Association between obstructive sleep apnea and cancer incidence in a large multicenter spanish cohort. Am J Respir Crit Care Med 2013; 187: 99-105.

7 Nieto FJ, Peppard PE, Young T, et al. Sleep-disordered breathing and cancer mortality: results from the Wisconsin Sleep Cohort Study. Am J Respir Crit Care Med 2012; 186: 190-194.

8 Almendros I, Montserrat JM, Ramirez J, et al. Intermittent hypoxia enhances cancer progression in a mouse model of sleep apnoea. Eur Respir J 2012; 39: 215-217.

9 Nanduri J, Yuan G, Kumar GK, et al. Transcriptional responses to intermittent hypoxia. Respir Physiol Neurobiol 2008; 164: 277-281.

10 Almendros I, Wang Y, Becker L, et al. Intermittent hypoxia-induced changes in tumor-associated macrophages and tumor malignancy in a mouse model of sleep apnea. Am J Respir Crit Care Med 2014; 189: 593-601.

11 Almendros I, Montserrat JM, Torres M, et al. Obesity and intermittent hypoxia increase tumor growth in a mouse model of sleep apnea. Sleep Med 2012; 13: 1254-1260.

12 Almendros I, Montserrat JM, Torres M, et al. Intermittent hypoxia increases melanoma metastasis to the lung in a mouse model of sleep apnea. Respir Physiol Neurobiol 2013; 186: 303-307.

13 Hakim F, Wang Y, Zhang SX, et al. Fragmented sleep accelerates tumor growth and progression through recruitment of tumor-associated macrophages and TLR4 signaling. Cancer Res 2014; 74: 1329-1337.

14 Martínez-García M-Á, Martorell-Calatayud A, Nagore E, et al. Association between sleep disordered breathing and aggressiveness markers of malignant cutaneous melanoma. Eur Respir J 2014; 43: 1661-1668.

15 Orgaz JL, Sanz-Moreno V. Emerging molecular targets in melanoma invasion and metastasis. Pigment Cell Melanoma Res 2013; 26: 39-57.

Eggermont AMM, Spatz A, Robert C. Cutaneous melanoma. Lancet 2014; 383: 816-827.

Hodis E, Watson IR, Kryukov GV, et al. A landscape of driver mutations in melanoma. Cell 2012; 150: 251-263. Mahabeleshwar GH, Byzova TV. Angiogenesis in melanoma. Semin Oncol 2007; 34: 555-565.

19 Wouters A, Pauwels B, Lardon F, et al. Review: implications of in vitro research on the effect of radiotherapy and chemotherapy under hypoxic conditions. Oncologist 2007; 12: 690-712.

20 Evans SM, Koch CJ. Prognostic significance of tumor oxygenation in humans. Cancer Lett 2003; 195: 1-16.

21 Semenza GL. Regulation of oxygen homeostasis by hypoxia-inducible factor 1. Physiology 2009; 24: 97-106.

22 Hong SS, Lee H, Kim KW. HIF-1 $\alpha$ : a valid therapeutic target for tumor therapy. Cancer Res Treat 2004; 36: 343-353.

23 Dewhirst MW, Cao Y, Moeller B. Cycling hypoxia and free radicals regulate angiogenesis and radiotherapy response. Nature Rev Cancer 2008; 8: 425-437. 
Toffoli S, Michiels C. Intermittent hypoxia is a key regulator of cancer cell and endothelial cell interplay in tumours. FEBS J 2008; 275: 2991-3002.

25 Belaidi E, Beguin PC, Levy P, et al. Prevention of HIF-1 activation and iNOS gene targeting by low-dose cadmium results in loss of myocardial hypoxic preconditioning in the rat. Am J Physiol Heart Circ Physiol 2008; 294: H901-H908.

26 Belaidi E, Joyeux-Faure M, Ribuot C, et al. Major role for hypoxia inducible factor-1 and the endothelin system in promoting myocardial infarction and hypertension in an animal model of obstructive sleep apnea. J Am Coll Cardiol 2009; 53: 1309-1317.

27 Yuan G, Nanduri J, Bhasker CR, et al. $\mathrm{Ca}^{+} /$calmodulin kinase-dependent activation of hypoxia inducible factor 1 transcriptional activity in cells subjected to intermittent hypoxia. J Biol Chem 2005; 280: 4321-4328.

28 Yuan G, Nanduri J, Khan S, et al. Induction of HIF-1 $\alpha$ expression by intermittent hypoxia: involvement of NADPH oxidase, $\mathrm{Ca}^{+}{ }^{+}$signaling, prolyl hydroxylases, and mTOR. J Cell Physiol 2008; 217: 674-685.

29 Lavie L. Obstructive sleep apnoea syndrome - an oxidative stress disorder. Sleep Med Rev 2003 ; 7: 35-51.

30 Troncoso Brindeiro CM, da Silva AQ, Allahdadi KJ, et al. Reactive oxygen species contribute to sleep apneainduced hypertension in rats. Am J Physiol Heart Circ Physiol 2007; 293: H2971-H2976.

31 Dopp JM, Philippi NR, Marcus NJ, et al. Xanthine oxidase inhibition attenuates endothelial dysfunction caused by chronic intermittent hypoxia in rats. Respiration 2011; 82: 458-467.

32 Norton CE, Jernigan NL, Kanagy NL, et al. Intermittent hypoxia augments pulmonary vascular smooth muscle reactivity to NO: regulation by reactive oxygen species. J Appl Physiol 2011; 111: 980-988.

33 Peng YJ, Nanduri J, Zhang X, et al. Endothelin-1 mediates attenuated carotid baroreceptor activity by intermittent hypoxia. J Appl Physiol 2012; 112: 187-196.

34 Ramond A, Godin-Ribuot D, Ribuot C, et al. Oxidative stress mediates cardiac infarction aggravation induced by intermittent hypoxia. Fundam Clin Pharmacol 2013; 27: 252-261.

35 Kaczmarek E, Bakker JP, Clarke DN, et al. Molecular biomarkers of vascular dysfunction in obstructive sleep apnea. PLoS One 2013; 8: e70559.

36 Lavie L, Kraiczi H, Hefetz A, et al. Plasma vascular endothelial growth factor in sleep apnea syndrome: effects of nasal continuous positive air pressure treatment. Am J Respir Crit Care Med 2002; 165: 1624-1628.

37 Gozal D, Lipton AJ, Jones KL. Circulating vascular endothelial growth factor levels in patients with obstructive sleep apnea. Sleep 2002; 25: 59-65.

38 Schulz R, Hummel C, Heinemann S, et al. Serum levels of vascular endothelial growth factor are elevated in patients with obstructive sleep apnea and severe nighttime hypoxia. Am J Respir Crit Care Med 2002; 165: 67-70.

39 Kalaria RN, Spoors L, Laude EA, et al. Hypoxia of sleep apnoea: cardiopulmonary and cerebral changes after intermittent hypoxia in rats. Respir Physiol Neurobiol 2004; 140: 53-62.

40 da Rosa DP, Forgiarini LF, Baronio D, et al. Simulating sleep apnea by exposure to intermittent hypoxia induces inflammation in the lung and liver. Mediators Inflamm 2012; 2012: 879419.

$41 \mathrm{Gu}$ CJ, Li M, Li QY, et al. Chronic intermittent hypoxia increases beta cell mass and activates the mammalian target of rapamycin/hypoxia inducible factor 1/vascular endothelial growth factor a pathway in mice pancreatic islet. Chin Med J 2013; 126: 2368-2373.

42 Lukyanova LD, Sukoyan GV, Kirova YI. Role of proinflammatory factors, nitric oxide, and some parameters of lipid metabolism in the development of immediate adaptation to hypoxia and HIF-1 $\alpha$ accumulation. Bull Exp Biol Med 2013; 154: 597-601.

43 Nikolaou V, Stratigos AJ. Emerging trends in the epidemiology of melanoma. Br J Dermatol 2014; 170: 11-19.

44 Peppard PE, Nieto FJ. Here come the sleep apnea-cancer studies. Sleep 2013; 36: 1409-1411. 